\title{
Elusive Niobium Alkyl Cations Related to Ethylene Polymerization
}

\author{
Helen M. Pritchard, ${ }^{\mathrm{a}, \mathrm{b}}$ Michel Etienne, ${ }^{\mathrm{a} *}$ Laure Vendier $^{\mathrm{a}}$ and G. Sean McGrady ${ }^{c}$
}

Laboratoire de Chimie de Coordination du CNRS, 205 Route de Narbonne, 31077 Toulouse Cedex

4, France, Department of Chemistry, King's College London, Strand, London, UK WC2R 2LS and

Department of Chemistry, University of New Brunswick, Fredericton, N. B., Canada E3B 6E2.

etienne@1cc-toulouse.fr

-Experimental details and characterization of compounds $2,3 \mathrm{a}, \mathrm{b}$

-Crystallographic data for $\left[\mathrm{Tp}^{\mathrm{Me} 2} \mathrm{NbMe}\left(\mathrm{OEt}_{2}\right)(\mathrm{MeC} \equiv \mathrm{CMe})\right]\left[\mathrm{BAr}_{\mathrm{f} 4}\right](2)$ 


\section{Experimental details}

All experiments were carried out under a dry dinitrogen atmosphere using either Schlenk tube or glove box techniques. Dichloromethane- $d_{2}$ was dried over molecular sieves, degassed by freezepump-thaw cycles and stored under dinitrogen. ${ }^{1} \mathrm{H},{ }^{13} \mathrm{C}$ and ${ }^{31} \mathrm{P}$ NMR spectra were obtained in dichloromethane- $d_{2}$ solutions on either a Brucker DPX 300 or a Brucker AMX 500 spectrometer using either Young's tap or a teflon disk sealed NMR tubes. Only pertinent ${ }^{1} J_{C H}$ are quoted in the ${ }^{13} \mathrm{C}$ spectra.

\section{$\left[\mathrm{Tp}^{\mathrm{Me} 2} \mathrm{NbMe}\left(\mathrm{OEt}_{2}\right)(\mathrm{MeC} \equiv \mathrm{CMe})\right]\left[\mathrm{BAr}_{\mathrm{f} 4}\right](2)$}

$\left[\mathrm{H}\left(\mathrm{Et}_{2} \mathrm{O}\right)_{2}\right]\left[\mathrm{BAr}_{\mathrm{f} 4}\right](55.5 \mathrm{mg}, 0.055 \mathrm{mmol})$ and $\mathrm{Tp}^{\mathrm{Me} 2} \mathrm{NbMe}_{2}(\mathrm{MeC} \equiv \mathrm{CMe})(\mathbf{1})(26 \mathrm{mg}, 0.055 \mathrm{mmol})$ were placed in an NMR tube and cooled $\left(-60^{\circ} \mathrm{C}\right.$, ethanol/liquid nitrogen bath). Cooled dichloromethane- $d_{2}\left(0.5 \mathrm{ml},-60^{\circ} \mathrm{C}\right)$ was added via syringe and the solution gently shaken to ensure all solids dissolved. The solution rapidly changed colour from yellow to orange and the evolution of gas over $10 \mathrm{~min}$. was noted. Alternatively, the tube was introduced in a pre-cooled probe of the NMR spectrometer, allowed to settle for at least $10 \mathrm{~min}$. at the given temperature before shimming and data acquisition were started. Single crystals of $\mathbf{2}$ could be obtained from a concentrated dichloromethane- $d_{2}$ solution in the NMR tube. Attempted C, H, N analysis on these thermally sensitive crystals gave unreliable results.

${ }^{1} \mathrm{H}$ NMR (300 MHz, $\left.213 \mathrm{~K}\right) \delta 7.79$ (br, $8 \mathrm{H}$, orthoH $\left.\mathrm{Ph}\left(\mathrm{CF}_{3}\right)_{2}\right), 7.59$ (br, $4 \mathrm{H}$, paraH $\left.\mathrm{Ph}\left(\mathrm{CF}_{3}\right)_{2}\right)$, 6.02, 5.96, 5.79 (s, $1 \mathrm{H}$ each, $\mathrm{Tp}^{\mathrm{Me} 2} \mathrm{CH}$ ), 4.69 (br, $\left.1 \mathrm{H}, \mathrm{Tp} * \mathrm{~B} H\right)$ 4.27, 4.13, 3.5, 3.4 (m, $1 \mathrm{H}$ each, $\left.\mathrm{O}\left(\mathrm{CH}_{2} \mathrm{Me}\right)_{2}\right) 3.25,2.40$ (s, $3 \mathrm{H}$ each, $M e \mathrm{C} \equiv \mathrm{CMe}$ ), 2.44, 2.39, 2.32, 2.21, 1.97, 1.60 (s, $3 \mathrm{H}$ each, $\left.\mathrm{Tp}^{\mathrm{Me} 2} \mathrm{Me}\right), 1.80(\mathrm{~s}, 3 \mathrm{H}, \mathrm{NbMe}), 1.35,0.92\left(\mathrm{~m}, 3 \mathrm{H}\right.$ each, $\left.\mathrm{O}\left(\mathrm{CH}_{2} \mathrm{Me}\right)_{2}\right)$.

${ }^{13} \mathrm{C}$ NMR $(75,5 \mathrm{MHz}, 213 \mathrm{~K}) \delta 254.8,245.8(\mathrm{~s}, \mathrm{Me} C \equiv C \mathrm{Me}), 161.6$ (q, ipsoCPh $\left.\left(\mathrm{CF}_{3}\right)_{2}, \mathrm{~J}_{\mathrm{BH}}=50 \mathrm{~Hz}\right)$, 151.5, 151.1, 149.4, 146.91, 146.87, 145.4 (s, $\left.\mathrm{Tp}^{\mathrm{Me} 2} C \mathrm{Me}\right), 134.5$ (br, orthoCPh $\left(\mathrm{CF}_{3}\right)_{2}$, $\left.{ }^{1} \mathrm{~J}_{\mathrm{CH}}=159 \mathrm{~Hz}\right), 128.6\left(\mathrm{q}\right.$, metaC $\left.\mathrm{Ph}\left(\mathrm{CF}_{3}\right)_{2}, \mathrm{~J}_{\mathrm{CF}}=30 \mathrm{~Hz}\right), 124.3\left(\mathrm{q}, C \mathrm{~F}_{3} \mathrm{~J}_{\mathrm{CF}}=272 \mathrm{~Hz}\right), 117.4$ (br, paraC $\left.\mathrm{Ph}\left(\mathrm{CF}_{3}\right)_{2}{ }^{1} \mathrm{~J}_{\mathrm{CH}}=164 \mathrm{~Hz}\right), 108.3,108.1,107.3\left(\mathrm{~s}, \mathrm{Tp}^{\mathrm{Me} 2} \mathrm{CH}\right), 74.9\left(\mathrm{~s}, \mathrm{NbCH}_{3}{ }^{1} \mathrm{~J}_{\mathrm{CH}}=121 \mathrm{~Hz}\right), 75.0,74.4$ (s, $\left.\mathrm{O}\left(\mathrm{CH}_{2} \mathrm{Me}\right)_{2}\right), 23.2,22.0$ (s, $\left.\mathrm{MeC} \equiv \mathrm{CMe}\right), 15.7,14.2,13.9,13.1,12.6,12.4$ (s, $\left.\mathrm{Tp}^{\mathrm{Me} 2} \mathrm{CMe}\right), 13.9$, $12.2\left(\mathrm{O}\left(\mathrm{CH}_{2} \mathrm{Me}\right)_{2}\right)$.

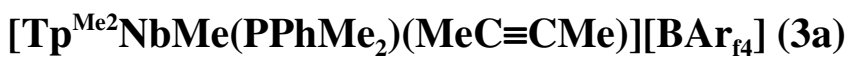

Cooled dichloromethane- $d_{2}\left(0.3 \mathrm{ml},-20^{\circ} \mathrm{C}\right.$, ethanol/liquid nitrogen bath) was added via syringe to a cooled NMR tube containing $\left[\mathrm{H}\left(\mathrm{Et}_{2} \mathrm{O}\right)_{2}\right]\left[\mathrm{BAr}_{\mathrm{f} 4}\right]\left(50.8 \mathrm{mg}, 0.050 \mathrm{mmol},-20^{\circ} \mathrm{C}\right)$; this was then cooled to $-60^{\circ} \mathrm{C}$. To a separate NMR tube containing $1\left(23.5 \mathrm{mg}, 0.050 \mathrm{mmol},-20^{\circ} \mathrm{C}\right)$ cooled dichloromethane- $d_{2}\left(0.2 \mathrm{ml},-20^{\circ} \mathrm{C}\right)$ was added, followed by an excess of cooled $\mathrm{PPhMe}_{2}(25 \mu 1$, $\left.0.176 \mathrm{mmol},-20^{\circ} \mathrm{C}\right)$. This yellow solution was added via a cannula to that of $\left[\mathrm{H}\left(\mathrm{Et}_{2} \mathrm{O}\right)_{2}\right]\left[\mathrm{BAr}_{\mathrm{f} 4}\right]$ and 
allowed to warm to $-20^{\circ} \mathrm{C}$. This temperature was maintained for $20 \mathrm{~min}$. and a gradual colour change from yellow to orange with the evolution of gas noted. On completion of the reaction the solution was cooled to $-60^{\circ} \mathrm{C}$, and analyzed by NMR spectroscopy.

${ }^{1} \mathrm{H}$ NMR $(300 \mathrm{MHz}, 213 \mathrm{~K}) \delta 7.82$ (br, $8 \mathrm{H}$, orthoH $\left.\mathrm{Ph}\left(\mathrm{CF}_{3}\right)_{2}\right), 7.60$ (br, $4 \mathrm{H}$, paraH $\left.\mathrm{Ph}\left(\mathrm{CF}_{3}\right)_{2}\right)$, 7.18, $6.70(\mathrm{~m}, \mathrm{PPhMe})$ 5.96, 5.88, 5.67 (s, $1 \mathrm{H}$ each, $\left.\mathrm{Tp}^{\mathrm{Me} 2} \mathrm{CH}\right), 3.52,2.57$ (s, $3 \mathrm{H}$ each, $M e \mathrm{C} \equiv \mathrm{CMe}$ ), 2.47, 2.43, 2.37, 1.88, 1.70, 1.63 (s, $3 \mathrm{H}$ each, $\mathrm{Tp}^{\mathrm{Me} 2} M e$ ), 1.68 (s, $3 \mathrm{H}, \mathrm{NbMe}$ ), 1.93, $1.25\left(\mathrm{~d}, 3 \mathrm{H}\right.$ each, $\left.\mathrm{PPh} M e_{2}\right)$

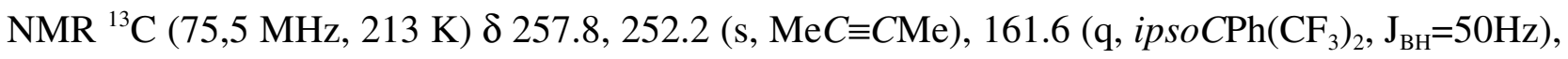
152.2, 152.1, 150.2, 147.6, 146.4, 145.6 (s, $\left.\mathrm{Tp}^{\mathrm{Me} 2} \mathrm{CMe}\right), 134.4$ (br, orthoCPh $\left.\left(\mathrm{CF}_{3}\right)_{2}\right)$, 131.0, 129.7, 128.8 (br, $\left.\mathrm{PP} h \mathrm{Me}_{2}\right), 128.2$ (q, metaCPh$\left.\left(\mathrm{CF}_{3}\right)_{2}\right), 124.2\left(\mathrm{q}, C \mathrm{~F}_{3}, \mathrm{~J}_{\mathrm{CF}}=272 \mathrm{~Hz}\right), 117.3$ (br, $\left.\operatorname{paraCPh}\left(\mathrm{CF}_{3}\right)_{2}\right), 108.6,108.3,107.1\left(\mathrm{~s}, \mathrm{Tp}^{\mathrm{Me} 2} \mathrm{CH}\right), 95.1\left(\mathrm{~s}, \mathrm{NbCH}_{3},{ }^{1} \mathrm{~J}_{\mathrm{CH}}=122 \mathrm{~Hz}\right), 24.0,23.95(\mathrm{~s}$, $M e \mathrm{C} \equiv \mathrm{CMe}), 15.7,15.4,14.8,13.0,12.7$ (s, $\mathrm{Tp}^{\mathrm{Me} 2} \mathrm{CMe}$ ), 13.9 (br, $\left.\mathrm{PPh} M e_{2}\right)$.

$\mathrm{NMR}{ }^{31} \mathrm{P}\left\{{ }^{1} \mathrm{H}\right\}(121,5 \mathrm{MHz}, 213 \mathrm{~K}) \delta-1.75\left(\mathrm{PPhMe}_{2}\right)$.

\section{$\left[\mathrm{Tp}^{\mathrm{Me} 2} \mathrm{NbMe}\left(\mathrm{PEt}_{3}\right)(\mathrm{MeC} \equiv \mathrm{CMe})\right]\left[\mathrm{BAr}_{\mathrm{f} 4}\right](3 \mathrm{~b})$}

Cooled degassed $\mathrm{PEt}_{3}\left(20 \mu 1,0.135 \mathrm{mmol},-60^{\circ} \mathrm{C}\right)$ was added via syringe to a dichloromethane- $d_{2}$ solution of $2\left(0.5 \mathrm{ml}, 0.05 \mathrm{mmol},-60^{\circ} \mathrm{C}\right)$ prepared as described above for complex 3a. This mixture was left for $18 \mathrm{hrs}$ at $-35^{\circ} \mathrm{C}$ to enable complete exchange between the diethyl ether and phosphine.

${ }^{1} \mathrm{H}$ NMR (500 MHz, $\left.233 \mathrm{~K}\right) \delta 7.75$ (br, $8 \mathrm{H}$, orthoH $\left.\mathrm{Ph}\left(\mathrm{CF}_{3}\right)_{2}\right), 7.58$ (br, $4 \mathrm{H}$, paraH $\left.\mathrm{Ph}\left(\mathrm{CF}_{3}\right)_{2}\right)$, 6.10, 5.91, 5.82 (s, $1 \mathrm{H}$ each, $\mathrm{Tp}^{\mathrm{Me} 2} \mathrm{CH}$ ), 3.40, 2.55 (s, $3 \mathrm{H}$ each, $M e \mathrm{C} \equiv \mathrm{C} M e$ ), 2.50, 2.39, 2.34, 2.23, 1.90, 1.62 (s, $3 \mathrm{H}$ each, $\mathrm{Tp}^{\mathrm{Me} 2} \mathrm{Me}$ ), 1.70 (br, $\left.3 \mathrm{H}, \mathrm{NbMe}\right), 1.78,1.52\left(\mathrm{~m}, \mathrm{P}\left(\mathrm{CH}_{2} \mathrm{Me}\right)_{3}\right) 0.62$ (m, $\left.\mathrm{P}\left(\mathrm{CH}_{2} \mathrm{Me}\right)_{3}\right)$

${ }^{13} \mathrm{C}\left\{{ }^{1} \mathrm{H}\right\} \operatorname{NMR}(125,8 \mathrm{MHz}, 233 \mathrm{~K}) \delta 161.6$ (q, ipsoC $\left.\mathrm{Ph}\left(\mathrm{CF}_{3}\right)_{2} \mathrm{~J}_{\mathrm{BH}}=50 \mathrm{~Hz}\right), 151.5,151.1,149.4$, 146.91, 146.87, 145.4 (s, Tp $\left.{ }^{\mathrm{Me} 2} C \mathrm{Me}\right), 134.5$ (br, orthoC $\left.\mathrm{Ph}\left(\mathrm{CF}_{3}\right)_{2}\right), 128.6$ (q, metaC $\mathrm{Ph}\left(\mathrm{CF}_{3}\right)_{2}$ $\left.\mathrm{J}_{\mathrm{CF}}=34 \mathrm{~Hz}\right), 124.3\left(\mathrm{q}, \mathrm{CF}_{3} \mathrm{~J}_{\mathrm{CF}}=272 \mathrm{~Hz}\right), 117.4\left(\mathrm{br}, \operatorname{paraC} \mathrm{Ph}\left(\mathrm{CF}_{3}\right)_{2}\right), 109.3,108.3,107.1(\mathrm{~s}$, $\left.\mathrm{Tp}^{\mathrm{Me} 2} \mathrm{CH}\right), 94.7\left(\mathrm{~s}, \mathrm{NbCH}_{3}\right), 24.42,24.37$ (s, $\left.\mathrm{MeC} \equiv \mathrm{CMe}\right), 15.8,15.6\left(\mathrm{~d}, \mathrm{P}\left(\mathrm{CH}_{2} \mathrm{Me}\right)_{3}\right), 16.5,15.2$, 14.7, 13.1, 12.9, 12.7 (s, $\left.\mathrm{Tp}^{\mathrm{Me} 2} \mathrm{CMe}\right), 6.7$ (br, $\left.\mathrm{P}\left(\mathrm{CH}_{2} \mathrm{Me}\right)_{3}\right)$.

$\mathrm{NMR}{ }^{31} \mathrm{P}\left\{{ }^{1} \mathrm{H}\right\}(208,4 \mathrm{MHz}, 233 \mathrm{~K}) \delta 15.3\left(\mathrm{~s}, P\left(\mathrm{CH}_{2} \mathrm{CH}_{3}\right)_{3}\right)$. 
Table 1. Crystal data and structure refinement for 2 .

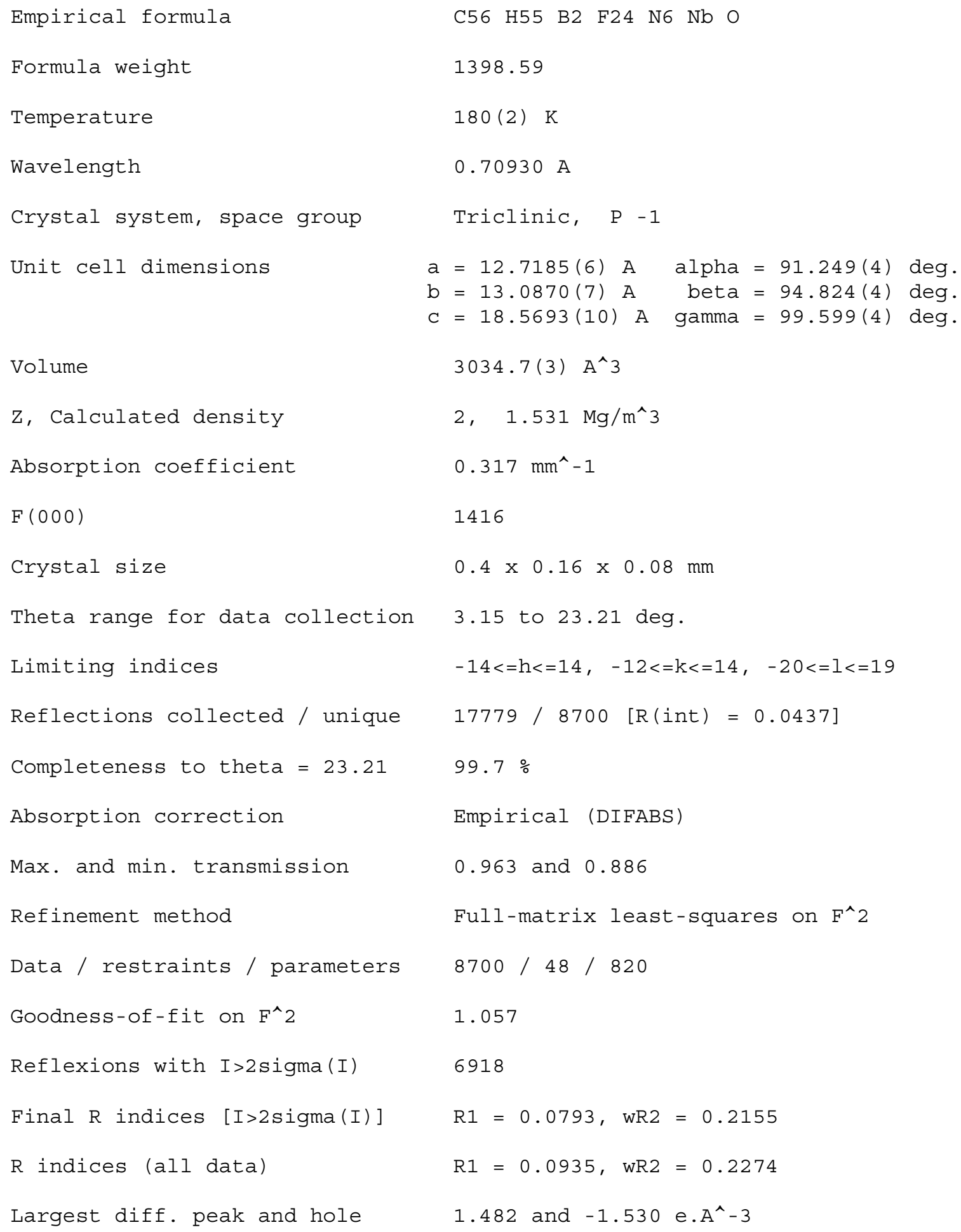


Data collection were collected at low temperature on an Xcalibur oxford Diffraction diffractometer using a graphite-monochromated Mo-K $\alpha$ radiation $(\lambda=0.71073)$ and equipped with an oxford Cryosystems Cryostream Cooler Device. The final unit cell parameters have been obtained by means of least-squares refinement performed on a set of 5000 well measured reflections. The structures have been solved by Direct Methods using SIR92[1], and refined by least-squares procedures on a $\mathrm{F}^{2}$ using the program SHELXL97[2]. The Atomic Scattering Factors were taken from International tables for X-Ray Crystallography [3]. Empirical absorbtion corrections were made [4]. Fluorine atoms of the anion were severely disordered and some of the $\mathrm{CF}_{3}$ groups were conveniently modeled over two different positions. Moreover, the complex cation was also disordered. C(18) and C(19) were found distributed over two different sites $C(18 A), C(19 A)$ and $C(18 B), C(19 B)$, with a site occupation ratio of 60/40. All hydrogen atoms were introduced in calculated positions and treated as riding model. All non-hydrogen atoms were anisotropically refined, and in the last cycles of refinement a weighting scheme was used, where weights are calculated from the following formula: $\mathrm{w}=1 /\left[\sigma^{2}\left(\mathrm{FO}^{2}\right)+(\mathrm{aP})^{2}+\mathrm{bP}\right]$ where $\mathrm{P}=\left(\mathrm{FO}^{2}+2 \mathrm{FC}^{2}\right) / 3$.

\section{References:}

$1 \quad$ SIR92 - A program for crystal structure solution. A.

Altomare, G. Cascarano, C. Giacovazzo and A. Guagliardi, J. Appl. Crystallogr. 1993, 26, 343-350.

2 SHELX97 [Includes SHELXS97, SHELXL97, CIFTAB] - Programs for Crystal Structure Analysis (Release 97-2). G. M. Sheldrick, Instit $t$ f $r$ Anorganische Chemie der Universit t, Tammanstrasse 4, D-3400 G ttingen, Germany, 1998 .

3 INTERNATIONAL tables for X-Ray crystallography, 1974, Vol IV, Kynoch press, Birmingham, England.

4 DIFABS - N. Walker and D. Stuart, Acta Crystallogr., Sect A 1983, 39, $158-166$. 
Table 2. Atomic coordinates $\left(\mathrm{x} 10^{\wedge} 4\right)$ and equivalent isotropic displacement parameters $\left(A^{\wedge} 2 \times 10^{\wedge} 3\right)$ for 2 .

$\mathrm{U}(\mathrm{eq})$ is defined as one third of the trace of the orthogonalized Uij tensor.

\begin{tabular}{|c|c|c|c|c|}
\hline & $\mathrm{x}$ & $\mathrm{Y}$ & $\mathrm{z}$ & $\mathrm{U}(\mathrm{eq})$ \\
\hline$C(1)$ & $6672(6)$ & $11511(6)$ & $8485(4)$ & $52(2)$ \\
\hline$C(2)$ & $7243(6)$ & $10723(6)$ & $8559(4)$ & $61(2)$ \\
\hline$C(3)$ & $6882(6)$ & $10012(5)$ & $7995(4)$ & $54(2)$ \\
\hline$C(4)$ & $6822(7)$ & $12504(7)$ & $8932(4)$ & $72(2)$ \\
\hline$C(5)$ & $7271(8)$ & $9040(6)$ & $7790(6)$ & $93(3)$ \\
\hline$C(6)$ & $5658(5)$ & $12253(5)$ & $5781(3)$ & $45(2)$ \\
\hline$C(7)$ & $6155(5)$ & $11635(5)$ & $5340(4)$ & $48(2)$ \\
\hline$C(8)$ & $6152(5)$ & $10711(5)$ & $5683(3)$ & $44(2)$ \\
\hline$C(9)$ & $5514(7)$ & $13343(6)$ & $5653(4)$ & $65(2)$ \\
\hline$C(10)$ & $6593(6)$ & $9788(6)$ & $5454(4)$ & $63(2)$ \\
\hline$C(11)$ & $2701(5)$ & $10099(5)$ & $7184(4)$ & $54(2)$ \\
\hline$C(12)$ & $2576(5)$ & $9049(5)$ & $7059(4)$ & $53(2)$ \\
\hline$C(13)$ & $3559(5)$ & $8825(5)$ & $6926(3)$ & $42(2)$ \\
\hline$C(14)$ & $1839(6)$ & $10734(7)$ & $7331(6)$ & $81(3)$ \\
\hline$C(15)$ & $3869(6)$ & $7823(5)$ & $6742(4)$ & $53(2)$ \\
\hline$C(16)$ & $3871(7)$ & $11008(7)$ & $8794(5)$ & $72(2)$ \\
\hline C (17) & $4277(9)$ & $11090(9)$ & $9535(5)$ & $96(3)$ \\
\hline$O(1)$ & $4041(4)$ & $11997(4)$ & $8432(3)$ & $63(1)$ \\
\hline$C(18 \mathrm{~A})$ & $3317(16)$ & $12651(13)$ & $8717(10)$ & $94(4)$ \\
\hline$C(19 A)$ & 3995 (15) & $13636(13)$ & $9036(9)$ & $94(4)$ \\
\hline$C(18 B)$ & $3980(20)$ & $12990(20)$ & 8812 (19) & $116(8)$ \\
\hline$C(19 B)$ & $2790(20)$ & $12980(20)$ & $8844(18)$ & $116(8)$ \\
\hline$C(20)$ & $3709(6)$ & $13310(6)$ & $7115(4)$ & $59(2)$ \\
\hline$C(21)$ & $3479(5)$ & $12585(5)$ & $6663(4)$ & $46(2)$ \\
\hline$C(22)$ & $3409(8)$ & $14336(6)$ & $7271(5)$ & $76(2)$ \\
\hline$C(23)$ & $2786(7)$ & $12303(7)$ & $5935(5)$ & $77(2)$ \\
\hline$C(30)$ & $10233(4)$ & $6300(4)$ & $6709(3)$ & $28(1)$ \\
\hline$C(31)$ & $10806(4)$ & $5714(4)$ & $6300(3)$ & $30(1)$ \\
\hline$C(32)$ & $11179(4)$ & $6046(4)$ & $5648(3)$ & $33(1)$ \\
\hline C ( 33$)$ & $11019(5)$ & $6989(5)$ & $5383(3)$ & $37(1)$ \\
\hline$C(34)$ & $10462(5)$ & $7590(4)$ & $5782(3)$ & $39(1)$ \\
\hline$C(35)$ & $10076(4)$ & $7247(4)$ & $6428(3)$ & $31(1)$ \\
\hline$C(36)$ & $11805(5)$ & $5388(5)$ & $5247(3)$ & $42(2)$ \\
\hline$C(37)$ & $10249(6)$ & $8615(5)$ & $5503(4)$ & $51(2)$ \\
\hline$C(40)$ & $9848(4)$ & $4807(4)$ & $7734(3)$ & $29(1)$ \\
\hline$C(41)$ & $10136(5)$ & $4577(5)$ & $8458(3)$ & $36(1)$ \\
\hline$C(42)$ & $10054(5)$ & $3556(5)$ & $8675(3)$ & $45(2)$ \\
\hline$C(43)$ & $9719(5)$ & $2731(5)$ & $8185(4)$ & $46(2)$ \\
\hline$C(44)$ & $9445(5)$ & $2946(5)$ & $7473(3)$ & $38(1)$ \\
\hline$C(45)$ & $9500(4)$ & $3961(4)$ & $7264(3)$ & $31(1)$ \\
\hline$C(46)$ & $9083(6)$ & $2079(5)$ & $6932(4)$ & $50(2)$ \\
\hline$C(50)$ & $10597(4)$ & $6840(4)$ & $8068(3)$ & $28(1)$ \\
\hline$C(51)$ & $10297(5)$ & $7711(4)$ & $8377(3)$ & $34(1)$ \\
\hline$C(52)$ & $11017(5)$ & $8420(4)$ & $8818(3)$ & $37(1)$ \\
\hline$C(53)$ & $12067(5)$ & $8285(5)$ & $8963(3)$ & $40(1)$ \\
\hline$C(54)$ & $12392(5)$ & $7441(5)$ & $8649(3)$ & $38(1)$ \\
\hline$C(55)$ & $11671(4)$ & $6728(4)$ & $8215(3)$ & $33(1)$ \\
\hline$C(57)$ & $13525(5)$ & $7261(6)$ & $8797(4)$ & $52(2)$ \\
\hline$C(60)$ & $8549(4)$ & $6115(4)$ & $7523(3)$ & $29(1)$ \\
\hline$C(61)$ & $7862(4)$ & $6170(4)$ & $6906(3)$ & $30(1)$ \\
\hline$C(62)$ & $6790(4)$ & $6229(4)$ & $6935(3)$ & $35(1)$ \\
\hline$C(63)$ & $6352(5)$ & $6220(4)$ & $7594(3)$ & $39(1)$ \\
\hline
\end{tabular}




\begin{tabular}{|c|c|c|c|c|}
\hline$C(64)$ & $7013(5)$ & $6139(5)$ & $8217(3)$ & $38(1)$ \\
\hline$C(65)$ & $8080(4)$ & $6087(4)$ & $8175(3)$ & $34(1)$ \\
\hline$C(67)$ & $6113(5)$ & $6328(5)$ & $6245(4)$ & $46(2)$ \\
\hline$C(24)$ & $5979(6)$ & $13458(5)$ & $7419(4)$ & $59(2)$ \\
\hline B (1) & $5462(5)$ & $9954(5)$ & $6878(4)$ & $37(2)$ \\
\hline$B(2)$ & $9814(5)$ & $6006(5)$ & $7503(3)$ & $28(1)$ \\
\hline $\mathrm{N}(1)$ & $5944(4)$ & $11309(4)$ & $7895(3)$ & $42(1)$ \\
\hline $\mathrm{N}(2)$ & $6091(4)$ & $10377(4)$ & $7594(3)$ & 41 (1) \\
\hline $\mathrm{N}(3)$ & $5657(4)$ & $10770(4)$ & $6302(2)$ & $35(1)$ \\
\hline $\mathrm{N}(4)$ & $5353(4)$ & $11731(4)$ & $6364(3)$ & $38(1)$ \\
\hline$N(5)$ & $4270(4)$ & $9730(3)$ & $6988(3)$ & $35(1)$ \\
\hline $\mathrm{N}(6)$ & $3749(4)$ & $10521(4)$ & $7146(3)$ & $47(1)$ \\
\hline$F(1)$ & $11848(4)$ & $5614(4)$ & $4551(2)$ & $67(1)$ \\
\hline$F(2)$ & $12805(3)$ & $5463(4)$ & $5523(2)$ & $61(1)$ \\
\hline$F(3)$ & $11381(4)$ & $4374(3)$ & $5245(3)$ & $70(1)$ \\
\hline$F(4)$ & $11043(5)$ & $9081(4)$ & $5156(4)$ & $120(2)$ \\
\hline$F(5)$ & $9439(5)$ & $8521(4)$ & $5018(3)$ & $114(2)$ \\
\hline$F(6)$ & $10114(6)$ & $9262(4)$ & $6009(3)$ & $105(2)$ \\
\hline$F(7)$ & $9718(6)$ & $2134(4)$ & $6395(3)$ & $113(2)$ \\
\hline$F(8)$ & $9149(5)$ & $1153(3)$ & $7176(3)$ & $96(2)$ \\
\hline$F(9)$ & $8158(5)$ & $2071(5)$ & $6630(5)$ & $160(4)$ \\
\hline$C(47)$ & $10292(7)$ & $3359(5)$ & $9455(5)$ & $76(3)$ \\
\hline$F(10 A)$ & $10994(9)$ & $2661(8)$ & $9502(5)$ & $102(2)$ \\
\hline$F(11 A)$ & $10746(10)$ & $4136(9)$ & $9888(5)$ & $102(2)$ \\
\hline$F(12 A)$ & $9486(9)$ & $2989(8)$ & $9805(5)$ & $102(2)$ \\
\hline$F(10 B)$ & $10434(15)$ & 2429 (11) & $9645(8)$ & $102(2)$ \\
\hline$F(11 B)$ & $11100(14)$ & $4107(14)$ & $9735(8)$ & $102(2)$ \\
\hline$F(12 B)$ & $9429(13)$ & $3542(13)$ & $9828(7)$ & $102(2)$ \\
\hline$C(56)$ & $10648(5)$ & $9298(5)$ & $9151(4)$ & $50(2)$ \\
\hline$F(13 A)$ & $11390(6)$ & $9990(6)$ & $9519(5)$ & $96(2)$ \\
\hline$F(14 A)$ & $10199(7)$ & $9895(6)$ & $8650(5)$ & $96(2)$ \\
\hline$F(15 A)$ & $9746(7)$ & $9065(7)$ & $9454(5)$ & $96(2)$ \\
\hline$F(13 B)$ & $11023(17)$ & $10228(10)$ & $8904(11)$ & $96(2)$ \\
\hline$F(14 B)$ & $9583(12)$ & $9276(16)$ & $9126(12)$ & $96(2)$ \\
\hline$F(15 B)$ & $10725(16)$ & $9228(13)$ & $9887(7)$ & $96(2)$ \\
\hline$F(16)$ & $13838(3)$ & $6685(4)$ & $8303(2)$ & $74(1)$ \\
\hline$F(17)$ & $14218(4)$ & $8148(5)$ & $8819(4)$ & $118(2)$ \\
\hline$F(18)$ & $13694(4)$ & $6838(6)$ & $9418(3)$ & $103(2)$ \\
\hline$C(66)$ & $6577(5)$ & $6115(5)$ & $8927(4)$ & $55(2)$ \\
\hline$F(19 A)$ & $6873(11)$ & $5303(8)$ & $9302(5)$ & $77(1)$ \\
\hline$F(20 A)$ & $7083(8)$ & $6956(8)$ & $9369(4)$ & $77(1)$ \\
\hline$F(21 A)$ & $5554(7)$ & $6128(10)$ & $8959(5)$ & $77(1)$ \\
\hline$F(19 B)$ & $7020(11)$ & $5642(10)$ & $9460(5)$ & $77(1)$ \\
\hline$F(20 B)$ & $6275(9)$ & $6979(8)$ & $9128(5)$ & $77(1)$ \\
\hline$F(21 B)$ & $5537(7)$ & $5504(9)$ & $8860(5)$ & $77(1)$ \\
\hline$F(22)$ & $6428(3)$ & $5859(3)$ & $5679(2)$ & $64(1)$ \\
\hline$F(23)$ & $5088(3)$ & $5927(3)$ & $6283(2)$ & $65(1)$ \\
\hline$F(24)$ & $6152(3)$ & $7318(3)$ & $6075(2)$ & $62(1)$ \\
\hline $\mathrm{Nb}(1)$ & $4647(1)$ & $12163(1)$ & $7366(1)$ & $44(1)$ \\
\hline
\end{tabular}


Table 3. Bond lengths [A] and angles [deg] for 2 .

\begin{tabular}{|c|c|}
\hline$C(1)-C(2)$ & $1.359(11)$ \\
\hline $\mathrm{C}(1)-\mathrm{N}(1)$ & $1.366(8)$ \\
\hline$C(1)-C(4)$ & $1.502(10)$ \\
\hline$C(2)-C(3)$ & $1.381(10)$ \\
\hline $\mathrm{C}(2)-\mathrm{H}(2)$ & 0.9500 \\
\hline $\mathrm{C}(3)-\mathrm{N}(2)$ & $1.357(8)$ \\
\hline$C(3)-C(5)$ & $1.494(11)$ \\
\hline $\mathrm{C}(4)-\mathrm{H}(4 \mathrm{~A})$ & 0.9800 \\
\hline $\mathrm{C}(4)-\mathrm{H}(4 \mathrm{~B})$ & 0.9800 \\
\hline $\mathrm{C}(4)-\mathrm{H}(4 \mathrm{C})$ & 0.9800 \\
\hline$C(5)-H(5 A)$ & 0.9800 \\
\hline $\mathrm{C}(5)-\mathrm{H}(5 \mathrm{~B})$ & 0.9800 \\
\hline $\mathrm{C}(5)-\mathrm{H}(5 \mathrm{C})$ & 0.9800 \\
\hline $\mathrm{C}(6)-\mathrm{N}(4)$ & $1.343(8)$ \\
\hline$C(6)-C(7)$ & $1.397(10)$ \\
\hline$C(6)-C(9)$ & $1.491(9)$ \\
\hline$C(7)-C(8)$ & $1.379(9)$ \\
\hline $\mathrm{C}(7)-\mathrm{H}(7)$ & 0.9500 \\
\hline$C(8)-N(3)$ & $1.362(8)$ \\
\hline$C(8)-C(10)$ & $1.483(10)$ \\
\hline $\mathrm{C}(9)-\mathrm{H}(9 \mathrm{~A})$ & 0.9800 \\
\hline$C(9)-H(9 B)$ & 0.9800 \\
\hline $\mathrm{C}(9)-\mathrm{H}(9 \mathrm{C})$ & 0.9800 \\
\hline $\mathrm{C}(10)-\mathrm{H}(10 \mathrm{~A})$ & 0.9800 \\
\hline $\mathrm{C}(10)-\mathrm{H}(10 \mathrm{~B})$ & 0.9800 \\
\hline $\mathrm{C}(10)-\mathrm{H}(10 \mathrm{C})$ & 0.9800 \\
\hline $\mathrm{C}(11)-\mathrm{N}(6)$ & $1.364(8)$ \\
\hline$C(11)-C(12)$ & $1.370(10)$ \\
\hline$C(11)-C(14)$ & $1.519(10)$ \\
\hline$C(12)-C(13)$ & $1.371(10)$ \\
\hline $\mathrm{C}(12)-\mathrm{H}(12)$ & 0.9500 \\
\hline$C(13)-N(5)$ & $1.361(8)$ \\
\hline$C(13)-C(15)$ & $1.474(9)$ \\
\hline $\mathrm{C}(14)-\mathrm{H}(14 \mathrm{~A})$ & 0.9800 \\
\hline $\mathrm{C}(14)-\mathrm{H}(14 \mathrm{~B})$ & 0.9800 \\
\hline $\mathrm{C}(14)-\mathrm{H}(14 \mathrm{C})$ & 0.9800 \\
\hline $\mathrm{C}(15)-\mathrm{H}(15 \mathrm{~A})$ & 0.9800 \\
\hline $\mathrm{C}(15)-\mathrm{H}(15 \mathrm{~B})$ & 0.9800 \\
\hline $\mathrm{C}(15)-\mathrm{H}(15 \mathrm{C})$ & 0.9800 \\
\hline$C(16)-C(17)$ & $1.424(12)$ \\
\hline$C(16)-O(1)$ & $1.464(9)$ \\
\hline $\mathrm{C}(16)-\mathrm{H}(16 \mathrm{~A})$ & 0.9900 \\
\hline $\mathrm{C}(16)-\mathrm{H}(16 \mathrm{~B})$ & 0.9900 \\
\hline $\mathrm{C}(17)-\mathrm{H}(17 \mathrm{~A})$ & 0.9800 \\
\hline $\mathrm{C}(17)-\mathrm{H}(17 \mathrm{~B})$ & 0.9800 \\
\hline $\mathrm{C}(17)-\mathrm{H}(17 \mathrm{C})$ & 0.9800 \\
\hline $\mathrm{O}(1)-\mathrm{C}(18 \mathrm{~A})$ & $1.478(15)$ \\
\hline$O(1)-C(18 B)$ & $1.48(2)$ \\
\hline $\mathrm{O}(1)-\mathrm{Nb}(1)$ & $2.186(5)$ \\
\hline$C(18 A)-C(19 A)$ & $1.51(2)$ \\
\hline $\mathrm{C}(18 \mathrm{~A})-\mathrm{H}(18 \mathrm{~A})$ & 0.9900 \\
\hline $\mathrm{C}(18 \mathrm{~A})-\mathrm{H}(18 \mathrm{~B})$ & 0.9900 \\
\hline $\mathrm{C}(19 \mathrm{~A})-\mathrm{H}(19 \mathrm{~A})$ & 0.9800 \\
\hline$C(19 A)-H(19 B)$ & 0.9800 \\
\hline $\mathrm{C}(19 \mathrm{~A})-\mathrm{H}(19 \mathrm{C})$ & 0.9800 \\
\hline$C(18 B)-C(19 B)$ & $1.52(2)$ \\
\hline $\mathrm{C}(18 \mathrm{~B})-\mathrm{H}(18 \mathrm{C})$ & 0.9900 \\
\hline $\mathrm{C}(18 \mathrm{~B})-\mathrm{H}(18 \mathrm{D})$ & 0.9900 \\
\hline$C(19 B)-H(19 D)$ & 0.9800 \\
\hline
\end{tabular}




\begin{tabular}{|c|c|}
\hline $\mathrm{C}(19 \mathrm{~B})-\mathrm{H}(19 \mathrm{E})$ & 0.9800 \\
\hline $\mathrm{C}(19 \mathrm{~B})-\mathrm{H}(19 \mathrm{~F})$ & 0.9800 \\
\hline$C(20)-C(21)$ & $1.234(10)$ \\
\hline$C(20)-C(22)$ & $1.484(11)$ \\
\hline $\mathrm{C}(20)-\mathrm{Nb}(1)$ & $2.104(8)$ \\
\hline $\mathrm{C}(20)-\mathrm{H}(20)$ & 1.0000 \\
\hline$C(21)-C(23)$ & $1.553(11)$ \\
\hline $\mathrm{C}(21)-\mathrm{Nb}(1)$ & $2.047(7)$ \\
\hline $\mathrm{C}(21)-\mathrm{H}(21)$ & 1.0000 \\
\hline $\mathrm{C}(22)-\mathrm{H}(22 \mathrm{~A})$ & 0.9800 \\
\hline $\mathrm{C}(22)-\mathrm{H}(22 \mathrm{~B})$ & 0.9800 \\
\hline $\mathrm{C}(22)-\mathrm{H}(22 \mathrm{C})$ & 0.9800 \\
\hline $\mathrm{C}(23)-\mathrm{H}(23 \mathrm{~A})$ & 0.9802 \\
\hline $\mathrm{C}(23)-\mathrm{H}(23 \mathrm{~B})$ & 0.9802 \\
\hline $\mathrm{C}(23)-\mathrm{H}(23 \mathrm{C})$ & 0.9802 \\
\hline$C(30)-C(35)$ & $1.394(8)$ \\
\hline$C(30)-C(31)$ & $1.395(8)$ \\
\hline$C(30)-B(2)$ & $1.642(8)$ \\
\hline$C(31)-C(32)$ & $1.390(8)$ \\
\hline $\mathrm{C}(31)-\mathrm{H}(31)$ & 0.9500 \\
\hline$C(32)-C(33)$ & $1.378(8)$ \\
\hline$C(32)-C(36)$ & $1.493(8)$ \\
\hline$C(33)-C(34)$ & $1.386(9)$ \\
\hline $\mathrm{C}(33)-\mathrm{H}(33)$ & 0.9500 \\
\hline$C(34)-C(35)$ & $1.389(8)$ \\
\hline$C(34)-C(37)$ & $1.506(9)$ \\
\hline $\mathrm{C}(35)-\mathrm{H}(35)$ & 0.9500 \\
\hline$C(36)-F(2)$ & $1.318(7)$ \\
\hline$C(36)-F(1)$ & $1.336(7)$ \\
\hline$C(36)-F(3)$ & $1.346(8)$ \\
\hline$C(37)-F(6)$ & $1.293(8)$ \\
\hline$C(37)-F(5)$ & $1.298(8)$ \\
\hline$C(37)-F(4)$ & $1.315(9)$ \\
\hline$C(40)-C(45)$ & $1.383(8)$ \\
\hline$C(40)-C(41)$ & $1.414(8)$ \\
\hline$C(40)-B(2)$ & $1.644(8)$ \\
\hline$C(41)-C(42)$ & $1.394(9)$ \\
\hline $\mathrm{C}(41)-\mathrm{H}(41)$ & 0.9500 \\
\hline$C(42)-C(43)$ & $1.383(9)$ \\
\hline$C(42)-C(47)$ & $1.492(10)$ \\
\hline$C(43)-C(44)$ & $1.385(9)$ \\
\hline $\mathrm{C}(43)-\mathrm{H}(43)$ & 0.9500 \\
\hline$C(44)-C(45)$ & $1.384(8)$ \\
\hline$C(44)-C(46)$ & $1.486(9)$ \\
\hline $\mathrm{C}(45)-\mathrm{H}(45)$ & 0.9500 \\
\hline$C(46)-F(9)$ & $1.258(9)$ \\
\hline$C(46)-F(8)$ & $1.316(8)$ \\
\hline$C(46)-F(7)$ & $1.331(9)$ \\
\hline$C(50)-C(51)$ & $1.389(8)$ \\
\hline$C(50)-C(55)$ & $1.403(8)$ \\
\hline$C(50)-B(2)$ & $1.642(8)$ \\
\hline$C(51)-C(52)$ & $1.388(8)$ \\
\hline $\mathrm{C}(51)-\mathrm{H}(51)$ & 0.9500 \\
\hline$C(52)-C(53)$ & $1.382(9)$ \\
\hline$C(52)-C(56)$ & $1.456(9)$ \\
\hline$C(53)-C(54)$ & $1.376(9)$ \\
\hline $\mathrm{C}(53)-\mathrm{H}(53)$ & 0.9500 \\
\hline$C(54)-C(55)$ & $1.386(8)$ \\
\hline$C(54)-C(57)$ & $1.502(9)$ \\
\hline $\mathrm{C}(55)-\mathrm{H}(55)$ & 0.9500 \\
\hline$C(57)-F(16)$ & $1.305(8)$ \\
\hline$C(57)-F(18)$ & $1.307(8)$ \\
\hline$C(57)-F(17)$ & $1.333(9)$ \\
\hline
\end{tabular}




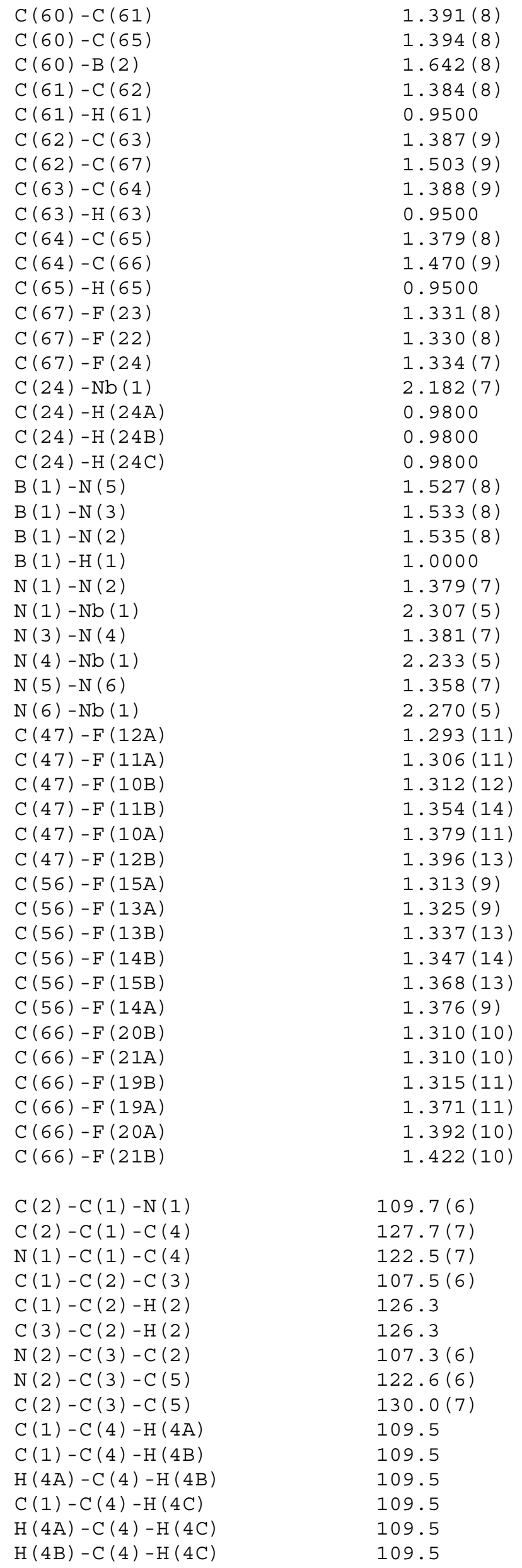




\begin{tabular}{|c|c|}
\hline$C(3)-C(5)-H(5 A)$ & 109.5 \\
\hline$C(3)-C(5)-H(5 B)$ & 109.5 \\
\hline $\mathrm{H}(5 \mathrm{~A})-\mathrm{C}(5)-\mathrm{H}(5 \mathrm{~B})$ & 109.5 \\
\hline$C(3)-C(5)-H(5 C)$ & 109.5 \\
\hline $\mathrm{H}(5 \mathrm{~A})-\mathrm{C}(5)-\mathrm{H}(5 \mathrm{C})$ & 109.5 \\
\hline $\mathrm{H}(5 \mathrm{~B})-\mathrm{C}(5)-\mathrm{H}(5 \mathrm{C})$ & 109.5 \\
\hline$N(4)-C(6)-C(7)$ & $109.8(6)$ \\
\hline$N(4)-C(6)-C(9)$ & $124.0(6)$ \\
\hline$C(7)-C(6)-C(9)$ & $126.1(6)$ \\
\hline$C(8)-C(7)-C(6)$ & $106.2(6)$ \\
\hline $\mathrm{C}(8)-\mathrm{C}(7)-\mathrm{H}(7)$ & 126.9 \\
\hline $\mathrm{C}(6)-\mathrm{C}(7)-\mathrm{H}(7)$ & 126.9 \\
\hline$N(3)-C(8)-C(7)$ & $107.9(6)$ \\
\hline$N(3)-C(8)-C(10)$ & $123.5(6)$ \\
\hline$C(7)-C(8)-C(10)$ & $128.6(6)$ \\
\hline $\mathrm{C}(6)-\mathrm{C}(9)-\mathrm{H}(9 \mathrm{~A})$ & 109.5 \\
\hline$C(6)-C(9)-H(9 B)$ & 109.5 \\
\hline $\mathrm{H}(9 \mathrm{~A})-\mathrm{C}(9)-\mathrm{H}(9 \mathrm{~B})$ & 109.5 \\
\hline$C(6)-C(9)-H(9 C)$ & 109.5 \\
\hline $\mathrm{H}(9 \mathrm{~A})-\mathrm{C}(9)-\mathrm{H}(9 \mathrm{C})$ & 109.5 \\
\hline $\mathrm{H}(9 \mathrm{~B})-\mathrm{C}(9)-\mathrm{H}(9 \mathrm{C})$ & 109.5 \\
\hline $\mathrm{C}(8)-\mathrm{C}(10)-\mathrm{H}(10 \mathrm{~A})$ & 109.5 \\
\hline $\mathrm{C}(8)-\mathrm{C}(10)-\mathrm{H}(10 \mathrm{~B})$ & 109.5 \\
\hline $\mathrm{H}(10 \mathrm{~A})-\mathrm{C}(10)-\mathrm{H}(10 \mathrm{~B})$ & 109.5 \\
\hline$C(8)-C(10)-H(10 C)$ & 109.5 \\
\hline $\mathrm{H}(10 \mathrm{~A})-\mathrm{C}(10)-\mathrm{H}(10 \mathrm{C})$ & 109.5 \\
\hline $\mathrm{H}(10 \mathrm{~B})-\mathrm{C}(10)-\mathrm{H}(10 \mathrm{C})$ & 109.5 \\
\hline$N(6)-C(11)-C(12)$ & $109.0(6)$ \\
\hline$N(6)-C(11)-C(14)$ & $123.6(6)$ \\
\hline$C(12)-C(11)-C(14)$ & $127.5(7)$ \\
\hline$C(11)-C(12)-C(13)$ & $107.1(6)$ \\
\hline $\mathrm{C}(11)-\mathrm{C}(12)-\mathrm{H}(12)$ & 126.5 \\
\hline $\mathrm{C}(13)-\mathrm{C}(12)-\mathrm{H}(12)$ & 126.5 \\
\hline$N(5)-C(13)-C(12)$ & $107.7(6)$ \\
\hline$N(5)-C(13)-C(15)$ & $122.6(6)$ \\
\hline$C(12)-C(13)-C(15)$ & $129.7(6)$ \\
\hline $\mathrm{C}(11)-\mathrm{C}(14)-\mathrm{H}(14 \mathrm{~A})$ & 109.5 \\
\hline $\mathrm{C}(11)-\mathrm{C}(14)-\mathrm{H}(14 \mathrm{~B})$ & 109.5 \\
\hline $\mathrm{H}(14 \mathrm{~A})-\mathrm{C}(14)-\mathrm{H}(14 \mathrm{~B})$ & 109.5 \\
\hline $\mathrm{C}(11)-\mathrm{C}(14)-\mathrm{H}(14 \mathrm{C})$ & 109.5 \\
\hline $\mathrm{H}(14 \mathrm{~A})-\mathrm{C}(14)-\mathrm{H}(14 \mathrm{C})$ & 109.5 \\
\hline $\mathrm{H}(14 \mathrm{~B})-\mathrm{C}(14)-\mathrm{H}(14 \mathrm{C})$ & 109.5 \\
\hline $\mathrm{C}(13)-\mathrm{C}(15)-\mathrm{H}(15 \mathrm{~A})$ & 109.5 \\
\hline $\mathrm{C}(13)-\mathrm{C}(15)-\mathrm{H}(15 \mathrm{~B})$ & 109.5 \\
\hline $\mathrm{H}(15 \mathrm{~A})-\mathrm{C}(15)-\mathrm{H}(15 \mathrm{~B})$ & 109.5 \\
\hline $\mathrm{C}(13)-\mathrm{C}(15)-\mathrm{H}(15 \mathrm{C})$ & 109.5 \\
\hline $\mathrm{H}(15 \mathrm{~A})-\mathrm{C}(15)-\mathrm{H}(15 \mathrm{C})$ & 109.5 \\
\hline $\mathrm{H}(15 \mathrm{~B})-\mathrm{C}(15)-\mathrm{H}(15 \mathrm{C})$ & 109.5 \\
\hline$C(17)-C(16)-O(1)$ & $113.3(8)$ \\
\hline $\mathrm{C}(17)-\mathrm{C}(16)-\mathrm{H}(16 \mathrm{~A})$ & 108.9 \\
\hline $\mathrm{O}(1)-\mathrm{C}(16)-\mathrm{H}(16 \mathrm{~A})$ & 108.9 \\
\hline $\mathrm{C}(17)-\mathrm{C}(16)-\mathrm{H}(16 \mathrm{~B})$ & 108.9 \\
\hline $\mathrm{O}(1)-\mathrm{C}(16)-\mathrm{H}(16 \mathrm{~B})$ & 108.9 \\
\hline $\mathrm{H}(16 \mathrm{~A})-\mathrm{C}(16)-\mathrm{H}(16 \mathrm{~B})$ & 107.7 \\
\hline $\mathrm{C}(16)-\mathrm{C}(17)-\mathrm{H}(17 \mathrm{~A})$ & 109.5 \\
\hline $\mathrm{C}(16)-\mathrm{C}(17)-\mathrm{H}(17 \mathrm{~B})$ & 109.5 \\
\hline $\mathrm{H}(17 \mathrm{~A})-\mathrm{C}(17)-\mathrm{H}(17 \mathrm{~B})$ & 109.5 \\
\hline $\mathrm{C}(16)-\mathrm{C}(17)-\mathrm{H}(17 \mathrm{C})$ & 109.5 \\
\hline $\mathrm{H}(17 \mathrm{~A})-\mathrm{C}(17)-\mathrm{H}(17 \mathrm{C})$ & 109.5 \\
\hline $\mathrm{H}(17 \mathrm{~B})-\mathrm{C}(17)-\mathrm{H}(17 \mathrm{C})$ & 109.5 \\
\hline$C(16)-O(1)-C(18 A)$ & $107.6(9)$ \\
\hline$C(16)-O(1)-C(18 B)$ & $121.7(16)$ \\
\hline$C(18 \mathrm{~A})-\mathrm{O}(1)-\mathrm{C}(18 \mathrm{~B})$ & $35.0(13)$ \\
\hline
\end{tabular}




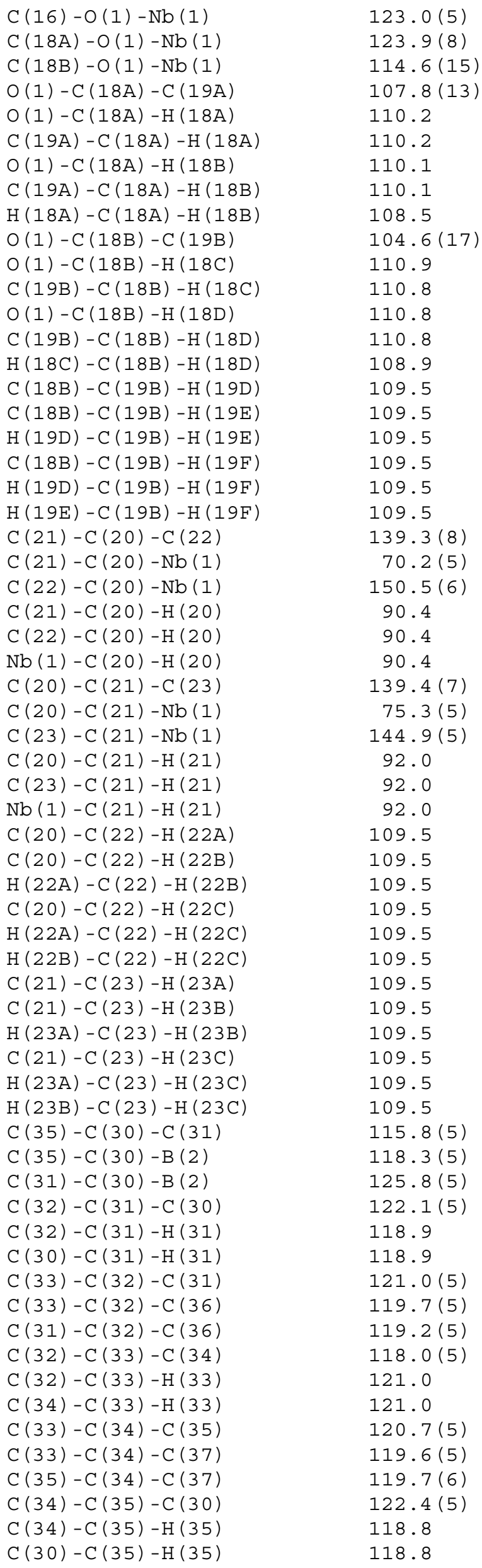

$123.9(8)$

$114.6(15)$

$107.8(13)$

110.2

110.2

110.1

110.1

108.5

$104.6(17)$

110.9

110.8

110.8

110.8

108.9

109.5

109.5

109.5

109.5

109.5

109.5

$139.3(8)$

$70.2(5)$

$150.5(6)$

90.4

90.4

90.4

$139.4(7)$

$75.3(5)$

$144.9(5)$

92.0

92.0

92.0

109.5

109.5

109.5

109.5

109.5

109.5

109.5

109.5

109.5

109.5

109.5

109.5

$115.8(5)$

$118.3(5)$

$125.8(5)$

122.1 (5)

118.9

118.9

$121.0(5)$

$119.7(5)$

$119.2(5)$

$118.0(5)$

121.0

121.0

120.7 (5)

$119.6(5)$

$119.7(6)$

$122.4(5)$

118.8

118.8 


\begin{tabular}{|c|c|}
\hline$F(2)-C(36)-F(1)$ & $106.2(5)$ \\
\hline$F(2)-C(36)-F(3)$ & $106.0(5)$ \\
\hline$F(1)-C(36)-F(3)$ & $105.2(5)$ \\
\hline$F(2)-C(36)-C(32)$ & $113.1(5)$ \\
\hline$F(1)-C(36)-C(32)$ & $113.4(5)$ \\
\hline$F(3)-C(36)-C(32)$ & $112.3(5)$ \\
\hline$F(6)-C(37)-F(5)$ & $108.9(7)$ \\
\hline$F(6)-C(37)-F(4)$ & $106.3(7)$ \\
\hline$F(5)-C(37)-F(4)$ & $102.9(7)$ \\
\hline$F(6)-C(37)-C(34)$ & $113.2(6)$ \\
\hline$F(5)-C(37)-C(34)$ & $113.0(6)$ \\
\hline$F(4)-C(37)-C(34)$ & $111.8(6)$ \\
\hline$C(45)-C(40)-C(41)$ & $115.9(5)$ \\
\hline$C(45)-C(40)-B(2)$ & $122.9(5)$ \\
\hline$C(41)-C(40)-B(2)$ & $120.9(5)$ \\
\hline$C(42)-C(41)-C(40)$ & $121.3(6)$ \\
\hline $\mathrm{C}(42)-\mathrm{C}(41)-\mathrm{H}(41)$ & 119.4 \\
\hline $\mathrm{C}(40)-\mathrm{C}(41)-\mathrm{H}(41)$ & 119.4 \\
\hline$C(43)-C(42)-C(41)$ & $121.1(6)$ \\
\hline$C(43)-C(42)-C(47)$ & $119.9(6)$ \\
\hline$C(41)-C(42)-C(47)$ & $118.9(6)$ \\
\hline$C(42)-C(43)-C(44)$ & $118.2(6)$ \\
\hline $\mathrm{C}(42)-\mathrm{C}(43)-\mathrm{H}(43)$ & 120.9 \\
\hline $\mathrm{C}(44)-\mathrm{C}(43)-\mathrm{H}(43)$ & 120.9 \\
\hline$C(45)-C(44)-C(43)$ & $120.6(6)$ \\
\hline$C(45)-C(44)-C(46)$ & $119.8(5)$ \\
\hline$C(43)-C(44)-C(46)$ & $119.6(6)$ \\
\hline$C(40)-C(45)-C(44)$ & $123.0(5)$ \\
\hline$C(40)-C(45)-H(45)$ & 118.5 \\
\hline $\mathrm{C}(44)-\mathrm{C}(45)-\mathrm{H}(45)$ & 118.5 \\
\hline$F(9)-C(46)-F(8)$ & $109.1(7)$ \\
\hline$F(9)-C(46)-F(7)$ & $105.2(8)$ \\
\hline$F(8)-C(46)-F(7)$ & $101.8(6)$ \\
\hline$F(9)-C(46)-C(44)$ & $113.9(6)$ \\
\hline$F(8)-C(46)-C(44)$ & $114.5(6)$ \\
\hline$F(7)-C(46)-C(44)$ & $111.2(6)$ \\
\hline$C(51)-C(50)-C(55)$ & $116.3(5)$ \\
\hline$C(51)-C(50)-B(2)$ & $124.5(5)$ \\
\hline$C(55)-C(50)-B(2)$ & $119.0(5)$ \\
\hline$C(52)-C(51)-C(50)$ & $121.8(5)$ \\
\hline $\mathrm{C}(52)-\mathrm{C}(51)-\mathrm{H}(51)$ & 119.1 \\
\hline $\mathrm{C}(50)-\mathrm{C}(51)-\mathrm{H}(51)$ & 119.1 \\
\hline$C(53)-C(52)-C(51)$ & $120.9(5)$ \\
\hline$C(53)-C(52)-C(56)$ & $119.7(6)$ \\
\hline$C(51)-C(52)-C(56)$ & $119.4(6)$ \\
\hline$C(54)-C(53)-C(52)$ & $118.6(5)$ \\
\hline $\mathrm{C}(54)-\mathrm{C}(53)-\mathrm{H}(53)$ & 120.7 \\
\hline $\mathrm{C}(52)-\mathrm{C}(53)-\mathrm{H}(53)$ & 120.7 \\
\hline$C(53)-C(54)-C(55)$ & $120.5(6)$ \\
\hline$C(53)-C(54)-C(57)$ & $120.2(5)$ \\
\hline$C(55)-C(54)-C(57)$ & $119.2(5)$ \\
\hline$C(54)-C(55)-C(50)$ & $121.9(5)$ \\
\hline $\mathrm{C}(54)-\mathrm{C}(55)-\mathrm{H}(55)$ & 119.0 \\
\hline $\mathrm{C}(50)-\mathrm{C}(55)-\mathrm{H}(55)$ & 119.0 \\
\hline$F(16)-C(57)-F(18)$ & $107.3(6)$ \\
\hline$F(16)-C(57)-F(17)$ & $104.6(6)$ \\
\hline$F(18)-C(57)-F(17)$ & $106.0(6)$ \\
\hline$F(16)-C(57)-C(54)$ & $114.0(5)$ \\
\hline$F(18)-C(57)-C(54)$ & $112.9(6)$ \\
\hline$F(17)-C(57)-C(54)$ & $111.4(6)$ \\
\hline$C(61)-C(60)-C(65)$ & $115.7(5)$ \\
\hline$C(61)-C(60)-B(2)$ & $123.6(5)$ \\
\hline$C(65)-C(60)-B(2)$ & $120.6(5)$ \\
\hline
\end{tabular}




$$
\begin{aligned}
& \mathrm{C}(62)-\mathrm{C}(61)-\mathrm{C}(60) \\
& \mathrm{C}(62)-\mathrm{C}(61)-\mathrm{H}(61) \\
& \mathrm{C}(60)-\mathrm{C}(61)-\mathrm{H}(61) \\
& C(61)-C(62)-C(63) \\
& C(61)-C(62)-C(67) \\
& C(63)-C(62)-C(67) \\
& \mathrm{C}(62)-\mathrm{C}(63)-\mathrm{C}(64) \\
& \mathrm{C}(62)-\mathrm{C}(63)-\mathrm{H}(63) \\
& \mathrm{C}(64)-\mathrm{C}(63)-\mathrm{H}(63) \\
& C(65)-C(64)-C(63) \\
& C(65)-C(64)-C(66) \\
& C(63)-C(64)-C(66) \\
& C(64)-C(65)-C(60) \\
& \mathrm{C}(64)-\mathrm{C}(65)-\mathrm{H}(65) \\
& \mathrm{C}(60)-\mathrm{C}(65)-\mathrm{H}(65) \\
& F(23)-C(67)-F(22) \\
& F(23)-C(67)-F(24) \\
& F(22)-C(67)-F(24) \\
& \mathrm{F}(23)-\mathrm{C}(67)-\mathrm{C}(62) \\
& F(22)-C(67)-C(62) \\
& F(24)-C(67)-C(62) \\
& \mathrm{Nb}(1)-\mathrm{C}(24)-\mathrm{H}(24 \mathrm{~A}) \\
& \mathrm{Nb}(1)-\mathrm{C}(24)-\mathrm{H}(24 \mathrm{~B}) \\
& \mathrm{H}(24 \mathrm{~A})-\mathrm{C}(24)-\mathrm{H}(24 \mathrm{~B}) \\
& \mathrm{Nb}(1)-\mathrm{C}(24)-\mathrm{H}(24 \mathrm{C}) \\
& \mathrm{H}(24 \mathrm{~A})-\mathrm{C}(24)-\mathrm{H}(24 \mathrm{C}) \\
& \mathrm{H}(24 \mathrm{~B})-\mathrm{C}(24)-\mathrm{H}(24 \mathrm{C}) \\
& \mathrm{N}(5)-\mathrm{B}(1)-\mathrm{N}(3) \\
& \mathrm{N}(5)-\mathrm{B}(1)-\mathrm{N}(2) \\
& \mathrm{N}(3)-\mathrm{B}(1)-\mathrm{N}(2) \\
& \mathrm{N}(5)-\mathrm{B}(1)-\mathrm{H}(1) \\
& \mathrm{N}(3)-\mathrm{B}(1)-\mathrm{H}(1) \\
& \mathrm{N}(2)-\mathrm{B}(1)-\mathrm{H}(1) \\
& \mathrm{C}(60)-\mathrm{B}(2)-\mathrm{C}(50) \\
& C(60)-B(2)-C(30) \\
& C(50)-B(2)-C(30) \\
& C(60)-B(2)-C(40) \\
& \mathrm{C}(50)-\mathrm{B}(2)-\mathrm{C}(40) \\
& \mathrm{C}(30)-\mathrm{B}(2)-\mathrm{C}(40) \\
& \mathrm{C}(1)-\mathrm{N}(1)-\mathrm{N}(2) \\
& \mathrm{C}(1)-\mathrm{N}(1)-\mathrm{Nb}(1) \\
& \mathrm{N}(2)-\mathrm{N}(1)-\mathrm{Nb}(1) \\
& \mathrm{C}(3)-\mathrm{N}(2)-\mathrm{N}(1) \\
& \mathrm{C}(3)-\mathrm{N}(2)-\mathrm{B}(1) \\
& \mathrm{N}(1)-\mathrm{N}(2)-\mathrm{B}(1) \\
& \mathrm{C}(8)-\mathrm{N}(3)-\mathrm{N}(4) \\
& \mathrm{C}(8)-\mathrm{N}(3)-\mathrm{B}(1) \\
& \mathrm{N}(4)-\mathrm{N}(3)-\mathrm{B}(1) \\
& \mathrm{C}(6)-\mathrm{N}(4)-\mathrm{N}(3) \\
& \mathrm{C}(6)-\mathrm{N}(4)-\mathrm{Nb}(1) \\
& \mathrm{N}(3)-\mathrm{N}(4)-\mathrm{Nb}(1) \\
& \mathrm{N}(6)-\mathrm{N}(5)-\mathrm{C}(13) \\
& \mathrm{N}(6)-\mathrm{N}(5)-\mathrm{B}(1) \\
& \mathrm{C}(13)-\mathrm{N}(5)-\mathrm{B}(1) \\
& \mathrm{N}(5)-\mathrm{N}(6)-\mathrm{C}(11) \\
& \mathrm{N}(5)-\mathrm{N}(6)-\mathrm{Nb}(1) \\
& \mathrm{C}(11)-\mathrm{N}(6)-\mathrm{Nb}(1) \\
& F(12 A)-C(47)-F(11 A) \\
& F(12 A)-C(47)-F(10 B) \\
& F(11 A)-C(47)-F(10 B) \\
& F(12 A)-C(47)-F(11 B) \\
& F(11 A)-C(47)-F(11 B) \\
& F(10 B)-C(47)-F(11 B)
\end{aligned}
$$




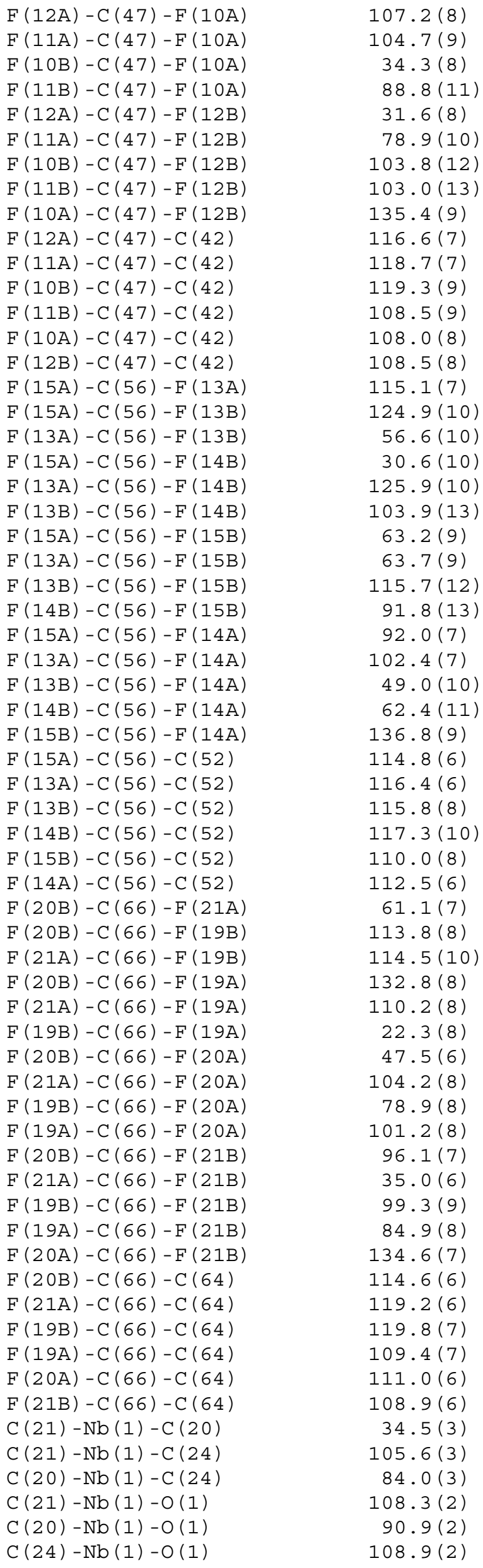

$104.7(9)$

$34.3(8)$

$88.8(11)$

$31.6(8)$

$78.9(10)$

$103.8(12)$

$103.0(13)$

$135.4(9)$

$116.6(7)$

$118.7(7)$

$119.3(9)$

$108.5(9)$

$108.0(8)$

$108.5(8)$

$115.1(7)$

$124.9(10)$

$56.6(10)$

$30.6(10)$

$125.9(10)$

$103.9(13)$

$63.2(9)$

$63.7(9)$

$115.7(12)$

$91.8(13)$

$92.0(7)$

$102.4(7)$

$49.0(10)$

$62.4(11)$

$136.8(9)$

$114.8(6)$

$116.4(6)$

$115.8(8)$

$117.3(10)$

$110.0(8)$

$112.5(6)$

$61.1(7)$

$113.8(8)$

$114.5(10)$

$132.8(8)$

$110.2(8)$

$22.3(8)$

$47.5(6)$

$104.2(8)$

$78.9(8)$

$101.2(8)$

$96.1(7)$

$35.0(6)$

$99.3(9)$

$84.9(8)$

$134.6(7)$

$114.6(6)$

$119.2(6)$

$119.8(7)$

$109.4(7)$

$111.0(6)$

$108.9(6)$

$34.5(3)$

$105.6(3)$

$84.0(3)$

$108.3(2)$

$90.9(2)$

$108.9(2)$ 


$$
\begin{array}{lc}
\mathrm{C}(21)-\mathrm{Nb}(1)-\mathrm{N}(4) & 84.6(2) \\
\mathrm{C}(20)-\mathrm{Nb}(1)-\mathrm{N}(4) & 108.5(2) \\
\mathrm{C}(24)-\mathrm{Nb}(1)-\mathrm{N}(4) & 82.8(2) \\
\mathrm{O}(1)-\mathrm{Nb}(1)-\mathrm{N}(4) & 158.55(19) \\
\mathrm{C}(21)-\mathrm{Nb}(1)-\mathrm{N}(6) & 85.3(2) \\
\mathrm{C}(20)-\mathrm{Nb}(1)-\mathrm{N}(6) & 113.6(3) \\
\mathrm{C}(24)-\mathrm{Nb}(1)-\mathrm{N}(6) & 158.3(2) \\
\mathrm{O}(1)-\mathrm{Nb}(1)-\mathrm{N}(6) & 84.5(2) \\
\mathrm{N}(4)-\mathrm{Nb}(1)-\mathrm{N}(6) & 79.53(19) \\
\mathrm{C}(21)-\mathrm{Nb}(1)-\mathrm{N}(1) & 163.0(2) \\
\mathrm{C}(20)-\mathrm{Nb}(1)-\mathrm{N}(1) & 162.0(3) \\
\mathrm{C}(24)-\mathrm{Nb}(1)-\mathrm{N}(1) & 82.5(3) \\
\mathrm{O}(1)-\mathrm{Nb}(1)-\mathrm{N}(1) & 82.31(19) \\
\mathrm{N}(4)-\mathrm{Nb}(1)-\mathrm{N}(1) & 81.51(18) \\
\mathrm{N}(6)-\mathrm{Nb}(1)-\mathrm{N}(1) & 82.50(19) \\
\end{array}
$$


Table 4. Anisotropic displacement parameters ( $A^{\wedge} 2 \times 10^{\wedge} 3$ ) for 2 . The anisotropic displacement factor exponent takes the form:

$-2 \mathrm{pi}^{\wedge} 2\left[\mathrm{~h}^{\wedge} 2 \mathrm{a}^{\star \wedge} 2 \mathrm{U} 11+\ldots+2 \mathrm{~h} \mathrm{k} \mathrm{a}^{*} \mathrm{~b} * \mathrm{U} 12\right]$

\begin{tabular}{|c|c|c|c|c|c|c|}
\hline & U11 & U2 2 & U33 & U23 & U13 & U12 \\
\hline$C(1)$ & $51(4)$ & $65(5)$ & $36(4)$ & $-4(3)$ & $-2(3)$ & $-1(4)$ \\
\hline$C(2)$ & $58(4)$ & $66(5)$ & $52(4)$ & $10(4)$ & $-18(4)$ & $2(4)$ \\
\hline$C(3)$ & $51(4)$ & $47(4)$ & $62(5)$ & $11(3)$ & $-15(4)$ & $7(3)$ \\
\hline$C(4)$ & $67(5)$ & $94(6)$ & $48(4)$ & $-24(4)$ & $-11(4)$ & $6(5)$ \\
\hline$C(5)$ & $93(7)$ & $48(5)$ & $130(8)$ & $-2(5)$ & $-59(6)$ & $28(5)$ \\
\hline$C(6)$ & $43(4)$ & $48(4)$ & $42(4)$ & $10(3)$ & $2(3)$ & $5(3)$ \\
\hline$C(7)$ & $41(4)$ & $61(4)$ & $40(4)$ & $10(3)$ & $5(3)$ & $-2(3)$ \\
\hline$C(8)$ & $34(3)$ & $51(4)$ & $42(4)$ & $-11(3)$ & $4(3)$ & $-5(3)$ \\
\hline C (9) & $74(5)$ & $59(5)$ & $66(5)$ & $27(4)$ & $14(4)$ & $17(4)$ \\
\hline$C(10)$ & $59(5)$ & $60(5)$ & $71(5)$ & $-15(4)$ & $28(4)$ & $2(4)$ \\
\hline$C(11)$ & $43(4)$ & $52(4)$ & $67(5)$ & $6(3)$ & $11(3)$ & $7(3)$ \\
\hline$C(12)$ & $39(4)$ & $58(4)$ & $57(4)$ & $7(3)$ & $1(3)$ & $-6(3)$ \\
\hline$C(13)$ & $43(4)$ & $40(3)$ & $39(3)$ & $7(3)$ & $-3(3)$ & $1(3)$ \\
\hline$C(14)$ & $46(4)$ & $72(6)$ & $125(8)$ & $0(5)$ & $27(5)$ & $7(4)$ \\
\hline$C(15)$ & $62(4)$ & $40(4)$ & $53(4)$ & $0(3)$ & $-7(3)$ & $-2(3)$ \\
\hline$C(16)$ & $75(6)$ & $72(5)$ & $70(6)$ & $21(4)$ & $16(4)$ & $7(4)$ \\
\hline$C(17)$ & $112(8)$ & $100(7)$ & $73(6)$ & $3(5)$ & $8(6)$ & $3(6)$ \\
\hline$O(1)$ & $82(4)$ & $64(3)$ & $48(3)$ & $5(2)$ & $23(3)$ & $21(3)$ \\
\hline$C(18 A)$ & $138(12)$ & $78(8)$ & $78(8)$ & $-2(6)$ & $25(8)$ & $48(8)$ \\
\hline$C(19 A)$ & $138(12)$ & $78(8)$ & $78(8)$ & $-2(6)$ & $25(8)$ & $48(8)$ \\
\hline$C(18 B)$ & $120(17)$ & $81(14)$ & $130(20)$ & $-16(13)$ & $-51(16)$ & $8(14)$ \\
\hline$C(19 B)$ & $120(17)$ & $81(14)$ & $130(20)$ & $-16(13)$ & $-51(16)$ & $8(14)$ \\
\hline$C(20)$ & $55(4)$ & $72(5)$ & $50(4)$ & $9(4)$ & $16(4)$ & $5(4)$ \\
\hline$C(21)$ & $39(3)$ & $39(4)$ & $64(4)$ & $10(3)$ & $17(3)$ & $7(3)$ \\
\hline$C(22)$ & $98(7)$ & $65(5)$ & $74(6)$ & $13(4)$ & $12(5)$ & $39(5)$ \\
\hline C (23) & $69(5)$ & $85(6)$ & $76(6)$ & $-13(5)$ & $-9(4)$ & $18(5)$ \\
\hline$C(30)$ & $22(3)$ & $35(3)$ & $24(3)$ & $-1(2)$ & $-5(2)$ & $3(2)$ \\
\hline$C(31)$ & $28(3)$ & $31(3)$ & $30(3)$ & $3(2)$ & $1(2)$ & $2(2)$ \\
\hline$C(32)$ & $25(3)$ & $44(3)$ & $27(3)$ & $-2(2)$ & $1(2)$ & $0(2)$ \\
\hline C (33) & $34(3)$ & $50(4)$ & $23(3)$ & $5(3)$ & $5(2)$ & $-2(3)$ \\
\hline$C(34)$ & $42(3)$ & $36(3)$ & $33(3)$ & $9(3)$ & $-5(3)$ & $-1(3)$ \\
\hline$C(35)$ & $35(3)$ & $33(3)$ & $27(3)$ & $1(2)$ & $-1(2)$ & $8(2)$ \\
\hline$C(36)$ & $37(4)$ & $58(4)$ & $29(3)$ & $1(3)$ & $3(3)$ & $5(3)$ \\
\hline$C(37)$ & $66(5)$ & $43(4)$ & $41(4)$ & $11(3)$ & $2(3)$ & $5(3)$ \\
\hline$C(40)$ & $26(3)$ & $37(3)$ & $25(3)$ & $4(2)$ & $2(2)$ & $8(2)$ \\
\hline$C(41)$ & $36(3)$ & $44(3)$ & $28(3)$ & $4(3)$ & $-3(2)$ & 8 (3) \\
\hline$C(42)$ & $51(4)$ & $45(4)$ & $39(4)$ & $13(3)$ & $-5(3)$ & $11(3)$ \\
\hline$C(43)$ & $45(4)$ & $39(3)$ & $53(4)$ & $14(3)$ & $-4(3)$ & $8(3)$ \\
\hline$C(44)$ & $35(3)$ & $40(3)$ & $39(3)$ & $2(3)$ & $2(3)$ & $8(3)$ \\
\hline$C(45)$ & $25(3)$ & $43(3)$ & $26(3)$ & $6(2)$ & $0(2)$ & $9(2)$ \\
\hline$C(46)$ & $59(4)$ & $39(4)$ & $51(4)$ & $0(3)$ & $-2(4)$ & $5(3)$ \\
\hline$C(50)$ & $32(3)$ & $31(3)$ & $22(3)$ & $4(2)$ & $5(2)$ & $9(2)$ \\
\hline$C(51)$ & $33(3)$ & $38(3)$ & $33(3)$ & $4(2)$ & $11(2)$ & $9(3)$ \\
\hline$C(52)$ & $45(4)$ & $36(3)$ & $30(3)$ & $-1(2)$ & $7(3)$ & $5(3)$ \\
\hline$C(53)$ & $44(4)$ & $43(3)$ & $30(3)$ & $-5(3)$ & $1(3)$ & $-1(3)$ \\
\hline$C(54)$ & $32(3)$ & $48(4)$ & $30(3)$ & $-2(3)$ & $-1(2)$ & $2(3)$ \\
\hline$C(55)$ & $35(3)$ & $35(3)$ & $27(3)$ & $-3(2)$ & $3(2)$ & $7(2)$ \\
\hline$C(57)$ & $39(4)$ & $67(5)$ & $48(4)$ & $-10(3)$ & $-6(3)$ & $13(3)$ \\
\hline$C(60)$ & $29(3)$ & $31(3)$ & $28(3)$ & $1(2)$ & $-1(2)$ & $7(2)$ \\
\hline$C(61)$ & $34(3)$ & $32(3)$ & $25(3)$ & $0(2)$ & $-2(2)$ & $8(2)$ \\
\hline$C(62)$ & $32(3)$ & $32(3)$ & $40(3)$ & $3(2)$ & $-7(3)$ & $10(2)$ \\
\hline$C(63)$ & $27(3)$ & $40(3)$ & $51(4)$ & $1(3)$ & $2(3)$ & $14(3)$ \\
\hline$C(64)$ & $34(3)$ & $45(3)$ & $38(3)$ & $4(3)$ & $6(3)$ & $12(3)$ \\
\hline
\end{tabular}




\begin{tabular}{|c|c|c|c|c|c|c|}
\hline$C(65)$ & $30(3)$ & $43(3)$ & $31(3)$ & $5(2)$ & $0(2)$ & $10(3)$ \\
\hline$C(67)$ & $37(4)$ & $51(4)$ & $50(4)$ & $1(3)$ & $-3(3)$ & $14(3)$ \\
\hline$C(24)$ & $65(5)$ & $42(4)$ & $64(5)$ & $-10(3)$ & $8(4)$ & $-5(3)$ \\
\hline$B(1)$ & $37(4)$ & $35(4)$ & $36(4)$ & $0(3)$ & $-3(3)$ & $5(3)$ \\
\hline B (2) & $25(3)$ & $35(3)$ & $24(3)$ & $0(3)$ & $-1(3)$ & $8(3)$ \\
\hline $\mathrm{N}(1)$ & $44(3)$ & $46(3)$ & $34(3)$ & $0(2)$ & $2(2)$ & $3(2)$ \\
\hline $\mathrm{N}(2)$ & $40(3)$ & $37(3)$ & $44(3)$ & $5(2)$ & $-5(2)$ & $5(2)$ \\
\hline$N(3)$ & $35(3)$ & $34(3)$ & $33(3)$ & $0(2)$ & $1(2)$ & $4(2)$ \\
\hline $\mathrm{N}(4)$ & $37(3)$ & $40(3)$ & $35(3)$ & $5(2)$ & $4(2)$ & $4(2)$ \\
\hline $\mathrm{N}(5)$ & $34(3)$ & $29(2)$ & $38(3)$ & $2(2)$ & $-1(2)$ & $2(2)$ \\
\hline $\mathrm{N}(6)$ & $38(3)$ & $40(3)$ & $60(4)$ & $3(2)$ & $7(3)$ & $2(2)$ \\
\hline$F(1)$ & $74(3)$ & $108(3)$ & $28(2)$ & $1(2)$ & $12(2)$ & $40(3)$ \\
\hline$F(2)$ & $36(2)$ & $103(3)$ & $46(2)$ & $-11(2)$ & $1(2)$ & $21(2)$ \\
\hline$F(3)$ & $71(3)$ & $56(3)$ & $86(3)$ & $-17(2)$ & $33(2)$ & $7(2)$ \\
\hline$F(4)$ & $134(5)$ & $73(3)$ & $167(6)$ & $73(4)$ & $69(5)$ & $23(3)$ \\
\hline$F(5)$ & $146(5)$ & $67(3)$ & $120(5)$ & $16(3)$ & $-74(4)$ & $31(3)$ \\
\hline$F(6)$ & $210(7)$ & $52(3)$ & $66(3)$ & $12(2)$ & $14(4)$ & $56(3)$ \\
\hline$F(7)$ & $178(6)$ & $77(3)$ & $73(3)$ & $-29(3)$ & $37(4)$ & $-14(4)$ \\
\hline$F(8)$ & $164(6)$ & $40(2)$ & $80(3)$ & $0(2)$ & $-1(3)$ & $8(3)$ \\
\hline F (9) & $103(5)$ & $122(5)$ & $239(9)$ & $-122(6)$ & $-108(5)$ & $52(4)$ \\
\hline$C(47)$ & $95(7)$ & $46(4)$ & $76(6)$ & $30(4)$ & $-38(5)$ & $-3(4)$ \\
\hline$F(10 A)$ & $135(5)$ & $119(4)$ & $50(2)$ & $44(3)$ & $-14(3)$ & $19(4)$ \\
\hline$F(11 A)$ & $135(5)$ & $119(4)$ & $50(2)$ & $44(3)$ & $-14(3)$ & $19(4)$ \\
\hline$F(12 A)$ & $135(5)$ & $119(4)$ & $50(2)$ & $44(3)$ & $-14(3)$ & $19(4)$ \\
\hline$F(10 B)$ & $135(5)$ & $119(4)$ & $50(2)$ & $44(3)$ & $-14(3)$ & $19(4)$ \\
\hline$F(11 B)$ & $135(5)$ & $119(4)$ & $50(2)$ & $44(3)$ & $-14(3)$ & $19(4)$ \\
\hline$F(12 B)$ & $135(5)$ & $119(4)$ & $50(2)$ & $44(3)$ & $-14(3)$ & $19(4)$ \\
\hline$C(56)$ & $59(4)$ & $38(4)$ & $54(4)$ & $-10(3)$ & $20(4)$ & $4(3)$ \\
\hline$F(13 A)$ & $107(3)$ & $71(3)$ & $117(4)$ & $-39(3)$ & $20(3)$ & $35(2)$ \\
\hline$F(14 A)$ & $107(3)$ & $71(3)$ & $117(4)$ & $-39(3)$ & $20(3)$ & $35(2)$ \\
\hline$F(15 A)$ & $107(3)$ & $71(3)$ & $117(4)$ & $-39(3)$ & $20(3)$ & $35(2)$ \\
\hline$F(13 B)$ & $107(3)$ & $71(3)$ & $117(4)$ & $-39(3)$ & $20(3)$ & $35(2)$ \\
\hline$F(14 B)$ & $107(3)$ & $71(3)$ & $117(4)$ & $-39(3)$ & $20(3)$ & $35(2)$ \\
\hline$F(15 B)$ & $107(3)$ & $71(3)$ & $117(4)$ & $-39(3)$ & $20(3)$ & $35(2)$ \\
\hline$F(16)$ & $42(2)$ & $128(4)$ & $56(3)$ & $-26(3)$ & $-3(2)$ & $35(2)$ \\
\hline$F(17)$ & $40(3)$ & $91(4)$ & $212(7)$ & $-31(4)$ & $-23(3)$ & $0(3)$ \\
\hline$F(18)$ & $73(3)$ & $205(7)$ & $51(3)$ & $27(3)$ & $4(2)$ & $76(4)$ \\
\hline$C(66)$ & $33(3)$ & $86(5)$ & $52(4)$ & $9(4)$ & $9(3)$ & $28(4)$ \\
\hline$F(19 A)$ & $75(3)$ & $118(4)$ & $46(2)$ & $0(3)$ & $22(2)$ & $34(3)$ \\
\hline$F(20 A)$ & $75(3)$ & $118(4)$ & $46(2)$ & $0(3)$ & $22(2)$ & $34(3)$ \\
\hline$F(21 A)$ & $75(3)$ & $118(4)$ & $46(2)$ & $0(3)$ & $22(2)$ & $34(3)$ \\
\hline$F(19 B)$ & $75(3)$ & $118(4)$ & $46(2)$ & $0(3)$ & $22(2)$ & $34(3)$ \\
\hline$F(20 B)$ & $75(3)$ & $118(4)$ & $46(2)$ & $0(3)$ & $22(2)$ & $34(3)$ \\
\hline$F(21 B)$ & $75(3)$ & $118(4)$ & $46(2)$ & $0(3)$ & $22(2)$ & $34(3)$ \\
\hline$F(22)$ & $69(3)$ & $81(3)$ & $46(2)$ & $-16(2)$ & $-20(2)$ & $35(2)$ \\
\hline$F(23)$ & $41(2)$ & $79(3)$ & $69(3)$ & $4(2)$ & $-18(2)$ & $6(2)$ \\
\hline$F(24)$ & 75 (3) & $48(2)$ & $63(3)$ & $12(2)$ & $-20(2)$ & $19(2)$ \\
\hline $\mathrm{Nb}(1)$ & $46(1)$ & $37(1)$ & $51(1)$ & $0(1)$ & $12(1)$ & $6(1)$ \\
\hline
\end{tabular}


Table 5. Hydrogen coordinates $\left(x 0^{\wedge} 4\right)$ and isotropic displacement parameters $\left(A^{\wedge} 2 \times 10^{\wedge} 3\right)$ for 2 .

\begin{tabular}{|c|c|c|c|c|}
\hline & $\mathrm{x}$ & $\mathrm{Y}$ & $\mathrm{z}$ & $\mathrm{U}(\mathrm{eq})$ \\
\hline $\mathrm{H}(2)$ & 7789 & 10670 & 8931 & 73 \\
\hline $\mathrm{H}(4 \mathrm{~A})$ & 7097 & 12383 & 9426 & 108 \\
\hline $\mathrm{H}(4 \mathrm{~B})$ & 6133 & 12744 & 8941 & 108 \\
\hline $\mathrm{H}(4 \mathrm{C})$ & 7333 & 13031 & 8719 & 108 \\
\hline $\mathrm{H}(5 \mathrm{~A})$ & 6719 & 8440 & 7859 & 139 \\
\hline $\mathrm{H}(5 \mathrm{~B})$ & 7925 & 8985 & 8095 & 139 \\
\hline $\mathrm{H}(5 \mathrm{C})$ & 7424 & 9056 & 7281 & 139 \\
\hline $\mathrm{H}(7)$ & 6438 & 11816 & 4894 & 58 \\
\hline $\mathrm{H}(9 \mathrm{~A})$ & 4846 & 13466 & 5839 & 97 \\
\hline $\mathrm{H}(9 \mathrm{~B})$ & 5484 & 13461 & 5132 & 97 \\
\hline $\mathrm{H}(9 \mathrm{C})$ & 6118 & 13820 & 5902 & 97 \\
\hline $\mathrm{H}(10 \mathrm{~A})$ & 7166 & 9669 & 5813 & 95 \\
\hline $\mathrm{H}(10 \mathrm{~B})$ & 6882 & 9906 & 4984 & 95 \\
\hline $\mathrm{H}(10 \mathrm{C})$ & 6024 & 9180 & 5413 & 95 \\
\hline $\mathrm{H}(12)$ & 1930 & 8566 & 7063 & 63 \\
\hline $\mathrm{H}(14 \mathrm{~A})$ & 2006 & 11078 & 7811 & 121 \\
\hline $\mathrm{H}(14 \mathrm{~B})$ & 1144 & 10275 & 7314 & 121 \\
\hline $\mathrm{H}(14 \mathrm{C})$ & 1812 & 11257 & 6964 & 121 \\
\hline $\mathrm{H}(15 \mathrm{~A})$ & 4104 & 7837 & 6252 & 80 \\
\hline $\mathrm{H}(15 \mathrm{~B})$ & 3254 & 7266 & 6763 & 80 \\
\hline $\mathrm{H}(15 \mathrm{C})$ & 4456 & 7697 & 7087 & 80 \\
\hline $\mathrm{H}(16 \mathrm{~A})$ & 3094 & 10734 & 8761 & 87 \\
\hline $\mathrm{H}(16 \mathrm{~B})$ & 4222 & 10505 & 8537 & 87 \\
\hline $\mathrm{H}(17 \mathrm{~A})$ & 5042 & 11381 & 9574 & 145 \\
\hline $\mathrm{H}(17 \mathrm{~B})$ & 4174 & 10400 & 9740 & 145 \\
\hline $\mathrm{H}(17 \mathrm{C})$ & 3893 & 11544 & 9801 & 145 \\
\hline $\mathrm{H}(18 \mathrm{~A})$ & 2909 & 12282 & 9092 & 112 \\
\hline $\mathrm{H}(18 \mathrm{~B})$ & 2802 & 12811 & 8323 & 112 \\
\hline $\mathrm{H}(19 \mathrm{~A})$ & 4466 & 13474 & 9448 & 141 \\
\hline $\mathrm{H}(19 \mathrm{~B})$ & 3531 & 14105 & 9200 & 141 \\
\hline $\mathrm{H}(19 \mathrm{C})$ & 4430 & 13972 & 8668 & 141 \\
\hline $\mathrm{H}(18 \mathrm{C})$ & 4312 & 13584 & 8542 & 139 \\
\hline $\mathrm{H}(18 \mathrm{D})$ & 4355 & 13021 & 9305 & 139 \\
\hline $\mathrm{H}(19 \mathrm{D})$ & 2445 & 12995 & 8353 & 174 \\
\hline $\mathrm{H}(19 \mathrm{E})$ & 2685 & 13599 & 9123 & 174 \\
\hline $\mathrm{H}(19 \mathrm{~F})$ & 2469 & 12358 & 9078 & 174 \\
\hline $\mathrm{H}(20)$ & 3147 & 12969 & 7419 & 71 \\
\hline $\mathrm{H}(21)$ & 2898 & 12227 & 6946 & 56 \\
\hline $\mathrm{H}(22 \mathrm{~A})$ & 3796 & 14857 & 6975 & 114 \\
\hline $\mathrm{H}(22 \mathrm{~B})$ & 3596 & 14531 & 7784 & 114 \\
\hline $\mathrm{H}(22 \mathrm{C})$ & 2637 & 14297 & 7157 & 114 \\
\hline $\mathrm{H}(23 \mathrm{~A})$ & 2217 & 12725 & 5891 & 116 \\
\hline $\mathrm{H}(23 \mathrm{~B})$ & 2467 & 11567 & 5920 & 116 \\
\hline $\mathrm{H}(23 \mathrm{C})$ & 3238 & 12440 & 5533 & 116 \\
\hline $\mathrm{H}(31)$ & 10945 & 5068 & 6474 & 36 \\
\hline $\mathrm{H}(33)$ & 11284 & 7219 & 4941 & 44 \\
\hline $\mathrm{H}(35)$ & 9691 & 7673 & 6687 & 38 \\
\hline $\mathrm{H}(41)$ & 10390 & 5129 & 8802 & 44 \\
\hline $\mathrm{H}(43)$ & 9678 & 2036 & 8332 & 55 \\
\hline $\mathrm{H}(45)$ & 9289 & 4083 & 6775 & 37 \\
\hline $\mathrm{H}(51)$ & 9581 & 7826 & 8284 & 41 \\
\hline $\mathrm{H}(53)$ & 12555 & 8763 & 9273 & 48 \\
\hline $\mathrm{H}(55)$ & 11912 & 6146 & 8010 & 39 \\
\hline $\mathrm{H}(61)$ & 8140 & 6167 & 6447 & 36 \\
\hline
\end{tabular}




$\begin{array}{lrrrr}H(63) & 5619 & 6268 & 7619 & 46 \\ H(65) & 8513 & 6029 & 8610 & 41 \\ H(24 A) & 5830 & 13959 & 7056 & 88 \\ H(24 B) & 6637 & 13201 & 7324 & 88 \\ H(24 C) & 6069 & 13796 & 7902 & 88 \\ H(1) & 5703 & 9302 & 6720 & 44\end{array}$

